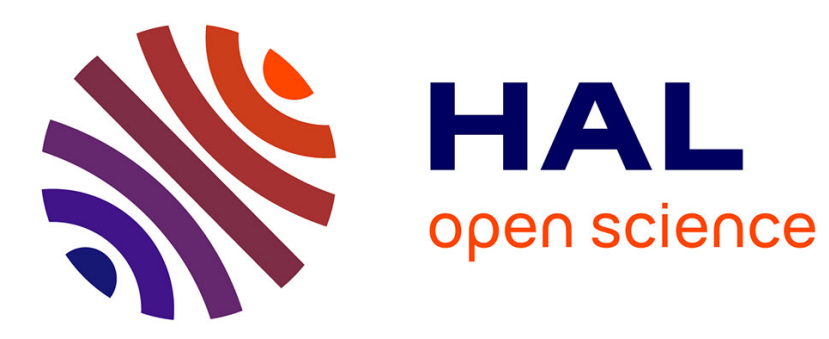

\title{
Bacterial suspensions under flow
}

Eric Clement, Anke Lindner, Carine Douarche, Harold Auradou

\section{To cite this version:}

Eric Clement, Anke Lindner, Carine Douarche, Harold Auradou. Bacterial suspensions under flow. The European Physical Journal. Special Topics, 2016, 225 (11-12), pp.2389 - 2406. 10.1140/epjst/e2016-60068-6 . hal-01404032

\section{HAL Id: hal-01404032 https://hal.sorbonne-universite.fr/hal-01404032}

Submitted on 28 Nov 2016

HAL is a multi-disciplinary open access archive for the deposit and dissemination of scientific research documents, whether they are published or not. The documents may come from teaching and research institutions in France or abroad, or from public or private research centers.
L'archive ouverte pluridisciplinaire HAL, est destinée au dépôt et à la diffusion de documents scientifiques de niveau recherche, publiés ou non, émanant des établissements d'enseignement et de recherche français ou étrangers, des laboratoires publics ou privés. 


\title{
Bacterial suspensions under flow
}

\author{
Eric Clement, ${ }^{\text {a }}$, Anke Lindner ${ }^{1}$, Carine Douarche ${ }^{2}$, and Harold Auradou ${ }^{3}$ \\ 1 PMMH, UMR7636 CNRS - ESPCI - University Pierre et Marie Curie - University Denis \\ Diderot, 10, rue Vauquelin 75005, Paris (FRANCE) \\ ${ }^{2}$ Laboratoire de Physique des Solides, Université Paris-Sud, CNRS UMR 8502, F-91405 \\ Orsay. \\ 3 Univ Paris-Sud, CNRS, F-91405. Lab FAST, Bât 502, Campus Univ, Orsay, F-91405 \\ (France).
}

\begin{abstract}
Fluids loaded with motile bacteria enter in the category of active matter, a new field currently developing at the convergence of biology, hydrodynamics and statistical physics. Such suspensions were shown recently to exhibit singular hydrodynamic transport properties. In this paper we review some recent results, either theoretical or experimental, on the active fluid rheology. We focus principally on bacteria suspensions and the objective is to provide the basis for understanding the emergence of the singular constitutive relations characterizing the macroscopic transport properties of such an active fluid under flow.
\end{abstract}

\section{Introduction}

Animals locomotion displays fascinating collective properties originating from interplay between individual self-propulsion and interactions among individuals in the group [1]. The notion of interaction is in itself a complex issue. It involves local ordering through contacts or longer range interactions mediated by the surrounding fluid and also, something more behavioural implying chemical sensing or cognitive decision. In spite of the large lexical variety describing these phenomena (mobs, herds, flocks, shoals, swarms), that applies to different animals moving in concert at different scales, it is only recently that physicists have elaborated the paradigmatic concept of active fluids. This notion enforces the statistical physics credo that more is different $[2]$ meaning that at some scales will emerge a unified physical entity ruled by macroscopic transport equations associated with constitutive relations. However, the nature of the emergent fields (stress, velocity, density), usually coupled with order parameters describing the local interaction processes, differs strongly from what is currently known for the standard physics of fluids. Theoretically, the phenomenological approaches highlight that due to the intrinsic symmetries of the microscopic interactions as well as the biological activity stemming from self-propulsion, many well-accepted notions describing matter at thermal equilibrium should be deeply revisited [3].

Fluids loaded with swimming micro-organisms are systems of choice to study these problems[4]. In recent studies, bacterial fluids were shown to revisit classical equilibrium properties of matter such as Brownian motion [5-7], Ficks law [8,9,11], spon-

\footnotetext{
a e-mail: eric.clement@upmc.fr
} 
taneous collective organization and low-Reynolds turbulence [13-16], and viscous response $[17,52,18,19]$. Experimentally these systems are rather easy to control and the recent advances in microfluidic technologies open the possibilities to fabricate environments where the biological activity and the motion in a flow can be assessed and controlled with great reliability [10-12]. Therefore, a true bottom/up approach of those active suspensions can be undertaken in order to establish the micro/macro passage on firm experimental ground.

The aim of the present course notes is to offer a synthetic presentation on bacterial suspension viewed as an active fluid and also to describe some recent experimental results illustrating the emergence of constitutive relations for bacterial suspensions under flow. This presentation is made in the context of recent advances in statistical mechanics of active suspensions developed from microscopic kinetic models hence providing a fundamental basis to describe the very peculiar rheology of active fluids at a macroscopic scale.

\section{The small force kingdom}

\subsection{Swimming strategies}

Due to their sizen, micro-organisms or active colloids moving in a fluid, cannot rely on inertia to propel autonomously. The fastest ones, can swim up to 10 times their body size in a second. Consequently, the corresponding Reynolds number is quite small $\left(10^{-5}-10^{-3}\right)$. The motility is thus governed by the Stokes equations (see the review by Winkler et al. [?] in this series ).

$$
\begin{gathered}
-\nabla P+\eta \nabla^{2} \mathbf{u}=0 \\
\nabla \cdot \mathbf{u}=0
\end{gathered}
$$

where $P$ and $\mathbf{u}$ are the fluid pressure and velocity. The Stokes equation for which time explicitly disappears, induces many important consequences for the propelling strategies. The most striking one is that any reciprocal motion implying the swimmer boundaries can not lead to net motion. This is called the "reciprocal theorems" or sometimes the "scallop theorem" [20]. To beat the time reversibility there are few strategies developed through evolution by the micro-organisms (For a review see for ex. Lauga et al. [21] or the book by Childress [22] and refs inside). For example, the production of a backwards undulation waves via the beating of a flagellum such as for sperms (see the review by Kaupp et al. [?] in this series ), produces a forwards motion. Generally, the production of waves, either tangential or normal to a body surface can be used for swimming at zero Reynolds. Such a scheme is developed by paramecia that can trigger metachronal surface waves of a cilia carpet in either direction, to move forwards and backwards. Also, some micro-organisms such as the mono-cellular algae Chlamydomonas Reinhardtii (eukaryotic organism, well studied by biologists) use two frontal flagella to swim a "breast-stroke" (see the review by Polin et al. [?] in this series ), meaning that the flagellum shape changes in different parts of the swimming cycle in order to induce a non-reciprocal motion. There is however another important case developed by many prokaryotes (essentially bacteria) lacking the possibility to activate internally a flagellum, contrarily to eukaryotic cells. Those micro-organisms use the rotation of helicoidal flagella attached to the body via a nano scale rotary motor [23]. The right association of the motor rotation and the flagellum helical handedness creates a thrust on the body coming from the back. It is important to see that, the case of Chlamydomonas Reinhardtii it is exactly the opposite, the body is pulled forwards by the flagella apparatus. Therefore both situations corresponds 
respectively to "pusher" and "puller' swimming strategies and we will develop in the following, important hydrodynamical consequences based on this fact.

\subsection{Scale of the driving forces}

If a micro-organism swims autonomously in a fluid, the necessary momentum conservation implies that there can not be any net force nor net torque acting on the fluid. Therefore, if there is a pushing (or pulling) force acting externally on the body there has to be an exactly opposite force acting on the propelling apparatus, essentially coming from the body drag resistance to motion. The same reasoning stands for torque. By law of action and reaction a thrust with a force $f$ means of course a similar force counteracting on the surrounding fluid (with the opposite sign). At this point, it is important to notice that for any fluid, there is an intrinsic force scale $F$ associated with the value of the viscosity $\eta_{0}$ and the mass density $\rho: F=\eta_{0}^{2} / \rho$. Therefore, motion at finite Reynolds number requires forces of typical magnitude:

$$
f=\operatorname{Re} F=\operatorname{Re} \eta_{0}^{2} / \rho
$$

Since the motion takes place at low Reynolds numbers, these forces are generically small with respect to the intrinsic fluid force scale (i.e. the "small force kingdom"). From the biological point of view, it is a source of energetic expense, crucial to assure motility and survival of the micro-organism. However, quantitatively, one must realize that the propulsive power is not likely to burden the global metabolic budget of the cell, only few percent [20]. Put in the pleasant phrasing of E.M.Purcell - in the midst of the 1970's oil crisis- a motile bacterium would feel "like driving a Datsun in Saudi Arabia". Indeed this has practical importance for the "minimal media" usually used as the surrounding fluid in which the bacterial suspension is prepared. This preparation is essentially composed of amino-acids and low sugar sources for energy. In response to this thinning regime, the bacteria would rather give-up splitting or making biofilms out of extracellular matrix production, two energetically expensive activities, but keep on swimming actively. This is how in practice controllable active suspensions are prepared. Let us consider for example, a bacterium like Escherichia Coli (E.coli) propelled via a bundle of half a dozen of rotating flagella at a velocity around $V_{0}=25 \mu \mathrm{m} / \mathrm{s}$. The body size is typically $a=2 \mu \mathrm{m}$. Therefore in water, the Reynolds number being $R e \approx 10^{-4}$ the propelling force is $f \approx 0.1 p N$. The energy needed to move fluid elements on the order of a bacterium size $l \approx 10 \mu m$ (including the flagella ) is :

$$
\sigma_{0}=f l \propto \eta_{0} V_{0} l^{2}
$$

This important energy scale is often called the "dipolar strength", a name which justification will be exposed in the next sub-chapter. From the mechanical point of view the dipolar strength is a central characterization of the organism motile activity. E.coli for example, yields $\sigma_{0} \approx 10^{-18} \mathrm{~J}$, a value overcoming by 2-3 three orders of magnitudes the thermal energy scale $\left(k_{B} T\right)$ and typically of the order of the Hamaker constants the characterizing the high energy surfaces. Consequently, very often bacteria stick to glassy surfaces unless some polymeric coating is used (then the Hamaker constant $\left.\approx k_{B} T \approx 10^{-21} \mathrm{~J}\right)$. For such bacteria, the propulsion velocity, is directly associated with the motor rotation rate: $V_{0} \propto \Omega$. The proportionality factor is a microscopic length scale depending only on the geometrical characteristics of the swimmer. It is important to see that the fluid viscosity scales-out of this picture provided that the motor rotation rate can be held constant by the bacterium activity. This is almost the case in nominal functioning conditions and thus, in a large range of viscosities, a bacterium is able to provide enough torque to maintain a fixed rotation rate and thus a constant swimming velocity (see Martinez et al.[24] and refs inside). 
(a)

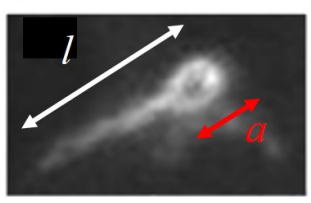

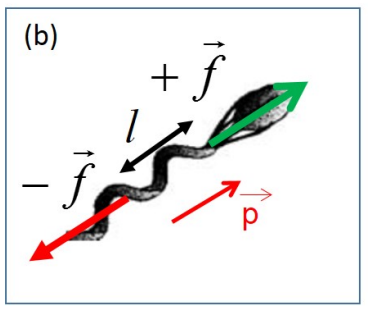

(c)

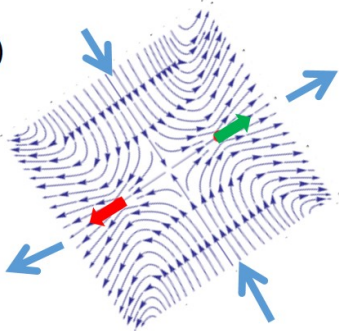

Fig. 1. Swimming E.coli bacterium (a) image of the swimming E.coli (ATCC9637) by fluorescent labelling of the body and the flagella. The body size is $a \approx 1.8 \mu \mathrm{m}$ and the total length, including the flagella bundle $l \approx 10 \mu \mathrm{m}$. (b) hydrodynamic representation of the bacterium as a "pusher" force dipole. (c) Resulting flow field created by the force dipole acting on the fluid. The blue arrows represent the main directions of the flow induced by the swimmer.

\subsection{Autonomous swimmers as force dipoles}

The fluid velocity field created by a moving bacterium is a-priori difficult to estimate in full details (see however, remarkable micro PIV measurements around microorganisms E.coli $[27]$ and Chlamidomonas $[25,26])$. As a generic feature for the Stoke's equations solutions for an isolated swimming bacterium, the flow field can be expanded asymptotically at large distances, as a series of force multipoles. At the lowest order of expansion, the dominant term is a force dipole (a stresslet), inducing in the bacterium reference frame, a flow field :

$$
\mathbf{u}=\epsilon \frac{\sigma_{0}}{8 \pi \eta_{0} r^{3}}\left(1-3 \cos \theta^{2}\right) \mathbf{r}
$$

and a pressure :

$$
P=\epsilon \frac{\sigma_{0}}{4 \pi r^{3}}\left(1-3 \cos \theta^{2}\right)
$$

where $\theta$ is the angle between the swimming direction and the radius vector $\mathbf{r}$, centred on the bacterium. The magnitude of the dipole is characterized by the dipolar strength $\sigma_{0}$. It is equivalent to two opposite forces of magnitude $f$ acting on the fluid along the motion direction and separated by a distance $l$; the centre is at a mid-distance from the points of application of the forces. Hence, $\sigma_{0}=f l$ and the two possible directions for the forces are given by the sign of $\epsilon$. For "pusher type" swimmers $\epsilon=-1$ and for pullers $\epsilon=1$. The structure of the fluid motion surrounding the swimming bacterium is a crucial element to keep in mind as it has important consequences on the emerging properties of the active fluid. For pusher swimmers, the induced fluid motion goes along the swimming direction in the front and is opposite in the tail. Also, the flow moves laterally towards the centre of the bacterium. Sometimes, such flow is called "extensile" (see Fig.1). For puller it is the reverse flow direction, and the situation is called "contractile'. Also, in some cases, the effective dipolar term may cancel and higher order multipolar contributions should come into play.

\subsection{Run \& tumble strategy for chemotaxis}

As wild-type species (i.e. not genetically modified), most micro-organisms organize their motility as regular changes of swimming directions. The aim of this wandering motion is to explore and harvest the environmental resources and eventually find 
the right ecological niche. At low Reynold's number such a directional change has to be accompanied with a shape modification suited to produce a torque on the body. The alternation of directed motion and directional changes is called "run \& tumble". For E.coli this strategy has been extensively studied. Following a biological signal, some motors changes their rotation direction provoking a partial or complete unbundling of the flagella; hence resulting in a reorientation of the swimming direction. In chemically homogeneous conditions, the run times and tumble times are distributed stochastically as Poisson variables with typical values $t_{\text {run }} \approx 1 s$ and $t_{\text {tmb }} \approx 0.1 s[23]$. Qualitatively, for the run and tumble strategy to make any sense, the bacteria has to move straight during the run time such as to overcome the molecular diffusion process. This argument provides a lower bound for the run length $\left(l_{\text {run }}>\sqrt{D_{m} t_{\text {run }}}\right)$ which means a minimal value : $l_{\text {run }}>D_{m} / V_{0}$, typically $100 \mu m$ for an E.coli, which is indeed the right magnitude observed. Interestingly, using tumbling mutants Saragosti et al. [28] have shown that the reorientation dynamics appears as a random walk on a unit sphere characterized by a rotational diffusion coefficient $D_{r}$. For a wild type, the resulting angular distribution for the redirection angles after tumbling $P(\theta)$ as measured originally by Berg [30], is a solution of this diffusion equation for $D_{r} t_{t m b} \approx 0.5$ $\left(D_{r}=3.5 r d^{2} / s\right.$ for a tumbling time $\left.t_{t m b}=0.14 s\right)$. The mean tumbling direction is $\left\langle\theta>\approx 68^{\circ}\right.$. In those conditions, the compound of run \& tumble motions induces on the long run, a diffusive motion characterized by a translational diffusivity:

$$
D_{t}=\frac{1}{3} \frac{V_{0}^{2} t_{r u n}}{1-<\cos \theta>} \approx \frac{V_{0}^{2} t_{r u n}}{6 D_{r} t_{t m b}}
$$

Thus following the trajectories of individual bacteria (or other micro-organisms) and averaging their mean-square displacements is a useful way to assess and characterize their activity. For non biased 3D isotropic motion, the mean-square displacement $<R^{2}>$ increases with the time-lag $\tau$, as :

$$
<R^{2}>=6 D_{t}\left(\tau-\tau_{c}\left(1-e^{-\tau / \tau_{c}}\right)\right)
$$

At long times, the diffusive motion : $\left\langle R^{2}\right\rangle=6 D_{t} \tau$, is recovered. However, at short times, a ballistic regime such that $\left\langle R^{2}\right\rangle=V_{0}^{2} \tau^{2}$, is observed and $D_{t}=\frac{1}{3} V_{0}^{2} \tau_{c}$ where $\tau_{c}$ is the crossover time. In practice, using a 2 parameter fit equation (as equ.(8)) the mean swimming velocity can be characterized as well as the long time diffusivity of the micro-organisms as a function of the environmental conditions (see examples of such measurements displayed in Fig (4)).

Note another useful complementary technique developed recently to characterize and monitor the mean activity of micro-organism suspensions. This technique called Differential Dynamic Microscopy (DDM), is based on the measurement of the spatio-temporal fluctuations of the average emitted light intensity and allows for high though-put measurements (see for ex. Martinez et al. and refs inside [29]).

For wild type E.coli, in nominal conditions, the translational diffusivity is $D_{t} \approx$ $100 \mu \mathrm{m}^{2} / \mathrm{s}$. This is to be related to the typical Brownian motion, computed for a $2 \mu \mathrm{m}$ diameter sphere (at $300 K): D_{B}=0.2 \mu \mathrm{m}^{2} / \mathrm{s}$, a typical value for inactive E.coli. The rotational diffusivity $\left(D_{r} \approx 3 r d^{2} / s\right)$ can be compared to the typical values undertaken by an elongated passive rod of size $l \approx 5-10 \mu m: D_{r}^{\text {rod }} \approx k_{B} T / \eta_{0} l^{3} \approx$ $10^{-2}-10^{-3} r d^{2} / s$. Therefore, in spite of the fact that it is not totally clear what should be the actual length contributing to the rotational viscous damping, for wild type species, the disorientation process is essentially controlled by the tumbling activity . Note that for some micro-organisms, the tumbling motion can be triggered by a mechanism different from a de-bundling process. For example, for marine bacteria Vibrio alginolyticus a flow triggered flagellar bucking is responsible for the change of direction [31] whereas for Chlamydomonas Reinhardtii a desynchronising process in 
the beat of the frontal flagella [32] triggers the reorientation (see the review by Polin et al. [?]in this series ) .

In a non uniform chemical environments, for example in a gradient of chemical attractants, micro-organisms adapt the run and tumble times to the result of the environmental exploration [23]. E.coli possesses a fine biochemical sensing equipments which trigger internal biological cascades and control the rate of reversal for the rotation of the nano-motor driving the flagella [33]. Thus, by enhancing the run time and/or diminishing the tumble time, the micro-organism is able to produce a net motion in the direction of gradients of attractants (or against if it is a repellent). This ability to follow chemical gradients is called chemotaxis [36]. For an extensive account of models describing mathematically the chemotactic behaviour see for example [34] and [35] and refs inside.

\subsection{Swimmers in interactions}

Swimming micro-organisms (pusher or puller) induce in their surrounding, a longrange flow field scaling as $\propto \sigma_{0} / r^{2}$. The effective interaction force decays rapidly with distance but may become significant when the approaching distance is of the order of few bacteria body size [37]. The sign of the interaction (attractive or repulsive) depends on the relative angular position and on the orientation between the swimmer. An extensive discussion and references can be found in the review by Lauga et al.[21]. Essentially, two side by side pusher swimmers are attracted whereas pullers are repelled. This can be qualitatively assessed from an inspection of the flow field as displayed for pusher swimmers in Fig.1(c) (for pullers, the flow lines are reversed). For micro-organisms swimming in line along the same direction, it is the opposite : pullers have the tendency to stay in lines while for pushers, this configuration is unstable as they repel. Also, since hydrodynamic shear induces a solid rotation of elongated objects, two pushers moving along the same direction but with some relative angle, will feel a torque that has the tendency to align them. Therefore they will finally swim, for a while, in the same direction. This is indeed an important element participating to the onset of collective motion as observed for concentrated suspensions of such micro-organisms (see infra).

Similarly, for bacteria swimming close to a solid surface, the pusher/puller distinction has a determinant influence. A bacterium moving close to a solid surface, for which the fluid boundary condition is a zero velocity, will "see" its hydrodynamic image. Consequently, pusher swimmers have the tendency to align along the surface and be attracted by hydrodynamic forces [47-49]. For puller swimmer, the perpendicular position is more favourable. Moreover, the elongated shape of most bacteria will further contribute to a nematic alignment with the surface simply by steric interactions (due to the impenetrability of the solid surface). Therefore, due to these effects, of both hydrodynamics and kinetic origins, pusher bacteria spend a long time swimming in the surface vicinity and thus their concentration displays a significant increase nearby solid surfaces.

In the context of active swimmers suspensions, is important to notice that the density of micro-organisms is often characterized by a volume fraction based on the body volume $\mathcal{V}_{b}$ :

$$
\phi=n \mathcal{V}_{b} \phi=n \mathcal{V}_{b}
$$

where $n$ is the number density and in the following, we use this definition to characterize the suspension concentration. For E.coli $\mathcal{V}_{b}=1 \mu \mathrm{m}^{3}$. However, to assess a situation that is really dilute, i.e. where interactions between bacteria can be seen as marginal, it is important to keep in mind that it is better to consider an excluded 


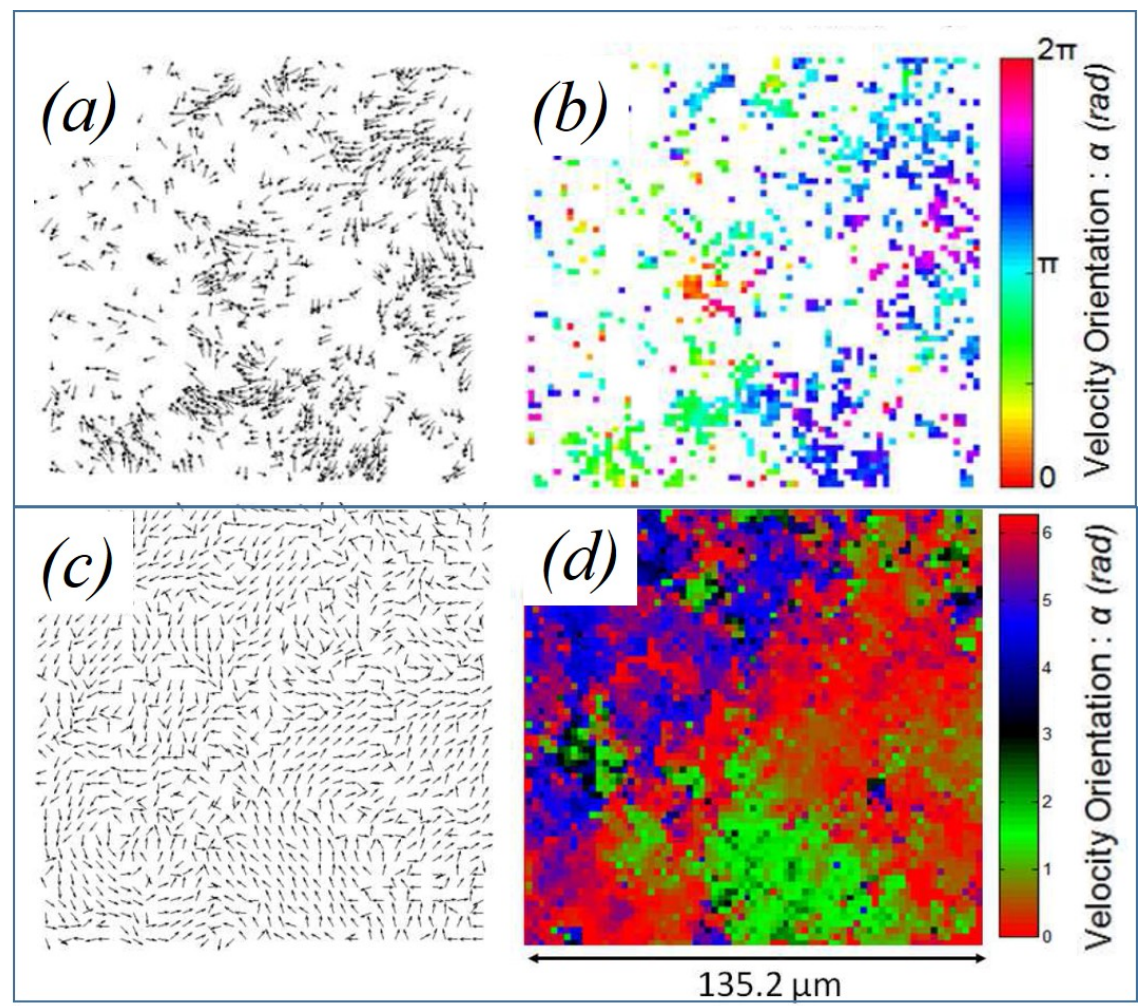

Fig. 2. Collective organization of E.coli bacteria (top panels volume fraction $\phi=8.010^{-3}$ and bottom panels $\phi=2.010^{-2}$ in absence of flow. The left pictures correspond to the bacteria velocity fields obtained by PIV measurements. The right pictures are the corresponding orientations $\alpha$ with respect to the horizontal direction and displayed in a colour map. Patches of similar colour of size much larger than a bacterium body size, witness of the large scale collective motion (See Gachelin et al. [16]).

volume based on a sphere with a diameter $l$ corresponding to the total swimmer size, including the flagella :

$$
\nu=n \frac{\pi}{6} l^{3} \approx \phi\left(\frac{l}{a}\right)^{3}
$$

with $a \approx \mathcal{V}_{b}^{1 / 3}$ the typical body size. The transition from dilute to semi-dilute regime is then occurring for the condition $\nu=O(1)$, which corresponds in practice, to very small values of $\phi$.

\subsection{Collective organization}

For bacteria suspensions, a natural aptitudes to undergo collective motion was identified by many experiments $[13-16]$ and also by numerical simulations [38-40]. From a theoretical point of view, the issue of collective organization for pushers is a natural outcome of the hydrodynamic processes triggered by generic interactions between the swimmers. It was addressed theoretically as a stability analysis of the hydrodynamic modes and complemented by numerical simulations to understand the dynamics beyond the linear regime $[41,38,39,42,40]$. An important outcome of these studies is that for rear-activated swimmers, a long range nematic ordering as well as a fully 
randomly oriented state are both unstable and essentially will lead to a mesoscopic collective motion $[41,40]$. When concentration is increased, the existence of a well define volume fraction triggering the onset of collective motion (as predicted for example by Subramanian and Koch [42]) is still debated. In a recent contribution Saintillan and Shelley [40] studied numerically the emergence of a correlated dynamics solely caused by hydrodynamic interactions (slender-body swimmers). For pushers, they show a progressive increase with concentration of the velocity correlation lengths when going from the dilute to the semi-dilute regime. This progressive appearance of collective motion without a clear threshold, was recovered experimentally with E.coli in 3D [16]. Qualitatively, the experimental observations witness a qualitative difference between the more dilute case showing small clusters of co-moving bacteria and the higher concentration where large scale collective motion appears (see Fig. 2). Note that in more confined environments (quasi 2D films or confined cells), the occurrence of a density threshold for collective motion was reported $[43,44]$. The reasons for this striking difference are still unclear but could be due to the nature of the hydrodynamics interaction in confined situations which become of shorter range because of the boundary screening effects.

\section{Statistical mechanics of active suspensions: the emergence of an "active fluid"}

\subsection{A simple kinetic model for dilute active suspensions : the "bacterial gas"}

There are different view points suited to handle the hydrodynamics of active fluids. First the macroscopic phenomenology approach as exposed for example in the review by Marchetti et al. [3]. In a nut shell, this type of approach identifies the relevant terms in the momentum and density transport equations stemming from general symmetry argument. It couples generically these fields with some relevant order parameters such as the polar direction of motion or the nematic alignment tensor. To close the theory, dynamical equations for the order parameter fields are added to the description. The second approach is more "bottom-up" or "micro/macro" in the statistical mechanics jargon, as it describes the microscopic behaviour and possible interactions between the swimmers to obtained by averaging, the macroscopic contributions to transport (see review by Marenduzzo et al. [?] in this series for a more complete picture). At the end, both approaches should provide similar macroscopic outcome, however the micro/macro path is clearly more difficult to handle when multiple interactions become significant. On the other hand, it provides testable predictions for the constitutive relations involving microscopic scale parameters.

Here we will only give an outlook for the simplest kinetic model aiming at establishing from the microscopic swimming properties, the viscosity of a dilute bacterial suspension in a flow. We will follow essentially the approach developed by Haines [53] and Saintillan [54] and provide qualitative and heuristic arguments to justify the generic form of the viscous response. For the more technical details, we recommend the reading those articles or for example, a summarized version developed in a review by Saintillan and Shelley [45].

In the dilute limit, interactions between bacteria can be neglected and the transport is modelled first by assuming that the local bacteria velocity is the vectorial sum of the swimming velocity $\mathbf{v}_{0}$ and the flow velocity $\mathbf{v}_{F}$ :

$$
\mathbf{v}(\mathbf{r})=\mathbf{v}_{0}+\mathbf{v}_{F}
$$

In a flow, the local shear rate induces a rotation of the body. The motion of the bacteria director $\mathbf{p}$ (the unit vector along the tail/head direction) can be represented 
at the most simple level, by a Fokker-Planck equation on the probability density for the director orientation $P(\mathbf{p})$ :

$$
\dot{P}+\nabla(\dot{\mathbf{p}} P)-D_{r} \nabla^{2} P=0
$$

where $D_{r}$ is an effective angular diffusivity.

Note here, the alternative and somehow more refined picture initially proposed by Subramanian and Koch [42] accounting explicitly for the run \& tumble process in equation (12). They argue that this process is essentially different from the continuous reorientation due to Brownian motion and introduce in equation (12) two terms, one for the orientation loss at the tumbling rate and a second one, for the orientation gain stemming from a kernel describing jumps in orientation. A generic form for this kernel is proposed to encompass situations ranging from random motion to persistent swimming. However in the simple and heuristic presentation of this review, we will only consider an effective rotation diffusion term accounting for all disorientation contributions either due to Brownian effects, to hydrodynamic instabilities or to active tumbling processes.

The orientation kinematics (in absence of noise ) of an elongated axisymmetric particle in a shear flow is usually modelled by the Bretherton-Jeffery equation.

$$
\dot{\mathbf{p}}=(\overline{\overline{1}}-\mathbf{p p})(\beta \overline{\bar{E}}+\overline{\bar{W}}) \mathbf{p}
$$

$\beta=\frac{r^{2}-1}{r^{2}+1}$ is the Bretherton constant characterizing the shape via the aspect ratio $r=l / a ; \overline{\bar{E}}$ and $\overline{\bar{W}}$ are respectively the shear and the rotation rate tensors. For a simple shear, characterized biy the shear rate $\dot{\gamma}$, the most salient outcome of such a model is an interplay between the shear rate aligning the bacteria along the flow direction at a time scale $\dot{\gamma}^{-1}$ and the active tumbling processes disorienting the bacteria at a time scale $D_{r}^{-1}$. The mean orientation of the bacteria under shear is thus controlled by a rotational Peclet number :

$$
P_{e}=\frac{\dot{\gamma}}{D_{r}}
$$

The mean desorientation angle (i.e. not along the flow) $\bar{\theta}$ is actually maximal when $P_{e}=O(1)$. At large $P_{e}$ a bacterium is oriented preferentially along the flow and the mean angle goes to zero with a scaling :

$$
\bar{\theta} \propto P_{e}^{-1}, P_{e}>>1
$$

similarly, at low $P_{e}$, the disorientation process due to the tumbling dominates and the mean orientation angle vanishes but with a linear scaling :

$$
\bar{\theta} \propto P_{e}, P_{e}<<1
$$

An illustration of this scaling behaviour is displayed in fig.3(c). The mean orientation angle is extracted from solving numerically a Bretherton-Jeffery equation with a random reorientation noise [51] which occurs at a Poissonian distribution of run times. The Bretherton constant used in this example is $\beta=0.6$.

For confined flows, bearing a non-homogeneous shear-gradient field a family of quasiperiodic trajectories comes out as a consequence of the shear gradient experienced by the bacteria [46]. This result indicates that the transport and dispersion properties of active suspensions are in this case non trivial. This is currently a very open problem which outcome turns out to be qualitatively different from the standard hydrodynamic transport of passive colloidal species [50] . 
(a)

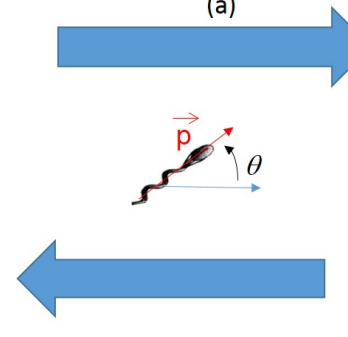

(b)

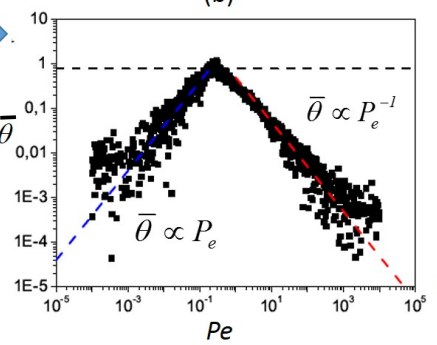

(c)

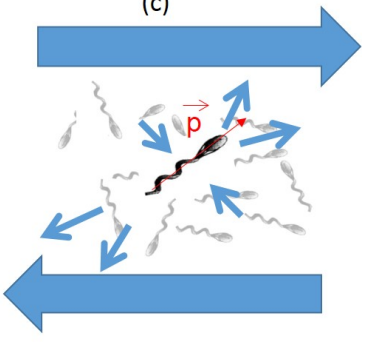

Fig. 3. Swimming and tumbling in a simple shear flow. (a) Schematic representation of a bacterium swimming at an angle $\theta$ with respect to the shear direction. (b) Result of numerical simulations of the Bretherton-Jeffery equation [51] in a simple shear and for a Poisson distribution of run times (average $t_{r u n}$ ) and random reorientation. The mean orientation angle $\bar{\theta}$ is displayed as a function of the Peclet number $P_{e}=\dot{\gamma} t_{\text {run }}$. The two regimes low shear $\left(\propto P_{e}\right)$ and high shear $\left(\propto P_{e}^{-1}\right)$ are displayed. The dotted horizontal line is $\bar{\theta}=\pi / 4$. (c) Schematics of the flow induced by a "pusher" swimmer oriented at an angle $\theta$ and acting on the shearing process. Qualitatively, when the swimmer is oriented with an angle $\pi / 4$, the motive action of the flow "helps" to shear, therefore the effective viscosity decreases.

In the following, we will review and discuss two situations. The first is an homogeneous shear which is the case of rheometric devices such as cone-plane geometries or Couette shear cell. The second case is a microfluidic Hele-shaw channel displaying a Poiseuille parabolic flow, a generic situation which opens the discussion on the more general issue of transport in confined environments such as capillary tubes or porous media.

\subsection{Viscosity of active suspensions in the dilute regime : the outcome of the kinetic models}

Following the scheme of calculation developed by Hinch and Leal [59] to understand the rheology of fibers, the shear stress contribution $\overline{\bar{\Sigma}}$ of the swimming dipole will add-up to the viscous contribution of the solvant. In the dilute regime, if Brownian motion is neglected, the stress tensor can be decomposed into two additive part :

$$
\overline{\bar{\Sigma}}=\overline{\bar{\Sigma}}_{p}+\overline{\bar{\Sigma}}_{a}
$$

where $\overline{\bar{\Sigma}}_{p}$ is a passive contribution coming from the particle drag and (ii) and $\overline{\bar{\Sigma}}_{a}$ is the active part which can be written, for a density $n$ of bacteria bearing a dipolar strength $\sigma_{0}$ :

$$
\bar{\Sigma}_{a} \propto n \sigma_{0} \int d \mathbf{p}(\overline{\bar{I}} / 3-\mathbf{p p}) P(\mathbf{p})
$$

where the average is taken over all the swimming orientations $P(\mathbf{p})$. The interpretation of the active shear stress contribution $\Sigma_{a}$ is straightforward as $n \sigma_{0}$ can be seen as a volumic density of energy to move the fluid at the scale of a bacterium size. Note that the prefactor's sign in equation (18) is positive for pullers and negative for pushers. The magnitude of the passive part $\Sigma_{p}$, due to the Stokes drag contribution, is $n \eta_{0} l^{3} \dot{\gamma}$ and corresponds to the energy loss per unit volume for the bacteria moving 
in the fluid at a velocity $\dot{\gamma} l$.

Now the question is to understand the relative contribution of both terms, active and passive on the effective viscosity to be added to the solvant viscosity $\eta_{0}$. For the passive part, the contribution is $\Sigma_{p} / \dot{\gamma} \propto \eta_{0} l^{3} \propto \eta_{0} \phi$. For the active part $\Sigma_{a}$, the contribution to viscosity is : $n \sigma_{0} / \dot{\gamma}$ and will depend on the effective mean orientation of the bacteria in the flow. Since at low shear rate, the bacteria still orient in average off the flow direction (see Fig.3) with a mean angle proportional to the shear rate (equ.(16)), the active contribution to the suspension viscosity $\eta_{a}=\frac{\Sigma_{a}}{\dot{\gamma}} \rightarrow \epsilon n \sigma_{0} / D_{r}$ converges to a constant value (cancellation of up and down $\dot{\gamma}$ 's). Note that the sign $\epsilon$ of this contribution to viscosity differs for "pusher" and "puller" swimmers. At low $\mathrm{Pe}$, the active contribution to viscosity is positive for pullers [52] and negative for pushers.

More quantitatively, using the previously exposed simple representation for the swimming kinematics, several theories [53-55] were quantitatively developed for the dilute regime. They predicted that the relative viscosity of the suspension $\eta$ depends on two dimensionless numbers via $\Psi\left(P_{e}, P_{e}^{b}\right)$ a dimensionless function and can be written as $:$

$$
\frac{\eta}{\eta_{0}}=1-\Psi\left(P_{e}, P_{e}^{b}\right) \phi
$$

where $P_{e}=\dot{\gamma} / D_{r}$ is the previously defined Peclet number and :

$$
P_{e}^{b}=\frac{V_{0}}{l D_{r}}
$$

is another dimensionless number involving the hydrodynamic time $: t_{c}=\frac{l}{V_{0}}$,i.e. the time for a bacterium to move over its own size. Also in their own framework, Subramanian and Koch [42] derived and expression for the linear viscous response of hydrodynamic modes. Their description eventually includes more details on the run/tumble dynamics dependence but comes out as essentially similar.

The outcome of the theories are (i) at high shear rate $\left(\dot{\gamma} t_{c}=P e / P_{e}^{b}>1\right)$ the active contribution to viscosity is negligible and a quasi-Newtonian plateau appears akin to suspensions of passive particles of the same shape; (ii) below a shear rate value $\dot{\gamma}_{c} t_{c}=O(1)$ the suspension viscosity is lower than the suspending fluid viscosity; (iii) at low shear rate $\left(P_{e}<<1\right)$, an active Newtonian plateau of viscosity $\eta_{p}$ appears such that:

$$
\frac{\eta_{p}}{\eta_{0}}=1+K\left(P_{e}^{b}\right) \phi
$$

where $K \propto\left(A+\epsilon B P_{e}^{b}\right) ; A$ and $B$ are positive constants with the same order of magnitude and solely determined by the micro-organism shape. Note that for pusher swimmer as bacteria $(\epsilon=-1), K$ may become negative for large values of $P_{e}^{b}$, yielding a volume fraction above which, the plateau viscosity $\eta_{p}$ becomes lower than the suspending fluid viscosity $\eta_{0}$. Note that Hatwalne et al. [56] also predicted using meanfield phenomenological hydrodynamic equations for active suspensions of pushers, the possibility for a viscosity decrease with concentration and the opposite behaviour for pullers.

\section{Viscosity of active bacterial suspensions : experiments}

Experimental evidence for viscosity reduction were brought for Bacillus subtilis [17] and $E$. coli [18]. It is only recently that a full rheological characterization (viscosity versus shear rate) was provided as a direct test for the kinetic theory for the viscous response under simple shear [19]. 


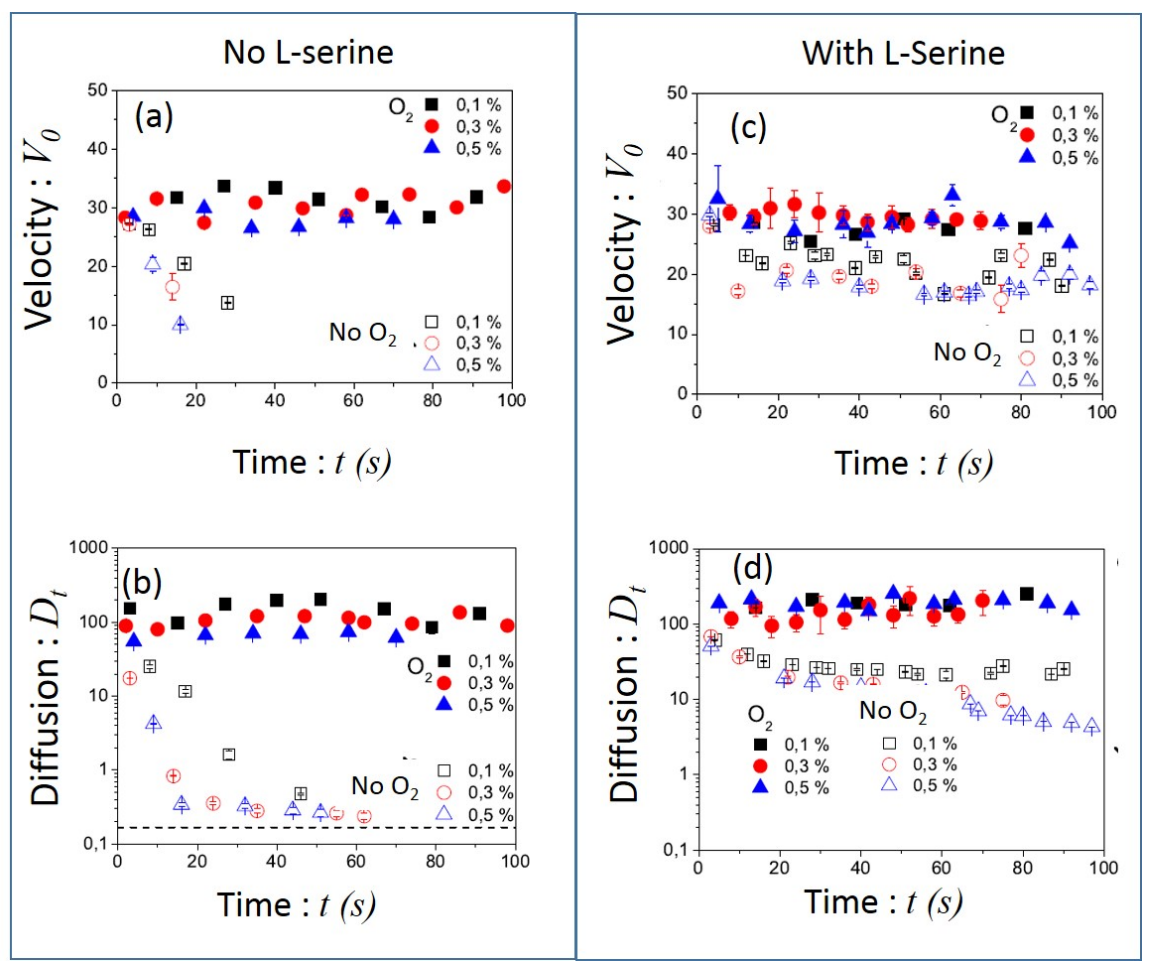

Fig. 4. Activity characterization of a E.coli (ATCC9637) suspension under different environmental conditions. From the mean square displacement of hundreds of tracks, the swimming velocities and the translation diffusivity coefficients are extracted (see equ.8), without (a),(b) and with (c),(d) L-serine. L-serine is an amino-acid that replaces the $\mathrm{O}_{2}$ respiration path. In absence of L-serine and $\mathrm{O}_{2}$, the bacteria asphyxiate and the activity drops down in a few minutes. However with L-serine, even in absence of $\mathrm{O}_{2}$, a significant activity is maintained [51].

\subsection{Maintaining the activity of a bacterial suspensions}

As for any biological material, the question of maintaining a constant activity in a controlled environment is a crucial issue to the completion of significant experimental work. To perform laboratory experiments with bacteria suspension, it is important to keep in mind that in a food rich medium, a population will double in typically $20 \min \left(\right.$ at $30^{\circ} \mathrm{C}$ ). Thus to avoid such a Malthusian growth, one needs to suspend the bacteria in a "minimal medium" suited to provide enough trace elements to maintain a swimming activity but discourage the cell growth. However, when deprived of $\mathrm{O}_{2}$ many bacteria strains such as E.coli can change their motility characteristics in a few minutes and can even "go to sleep"[58]. However, it is worth noticing that in soft micro-fluidic devices made of PDMS, gases like $\mathrm{O}_{2}$ can go through the polymeric structure. So in a large range of concentration, bacteria can access $\mathrm{O}_{2}$ in sufficient amount. Note also that some species such as Bacillus subtilis are quite sensitive to $\mathrm{O}_{2}$ shortage and for this reason, are delicate to handle in microfluidic devices even in PDMS. In the following, we will discuss essentially of E.coli strains.

To characterize the activity in various environments, by tracking hundreds of trajectories, the mean square displacement was computed as a function of time and the corresponding velocities $V_{0}$ as well as the translational velocities $D_{t}$ are reported in Fig. 4. To keep a constant activity in a closed environment, L-serine can be added 
(a)

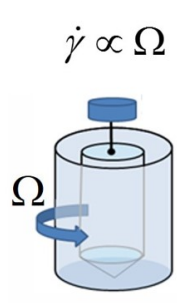

(b)

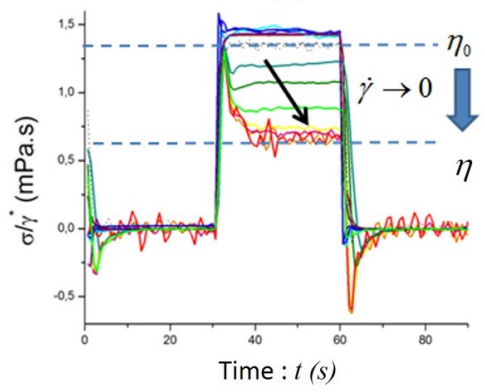

(c)

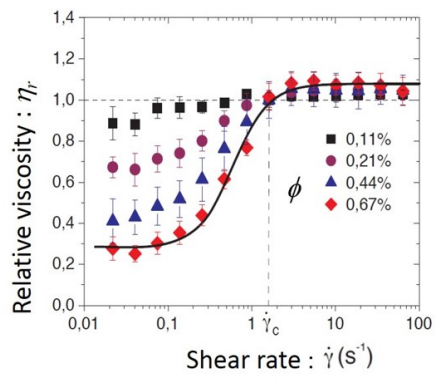

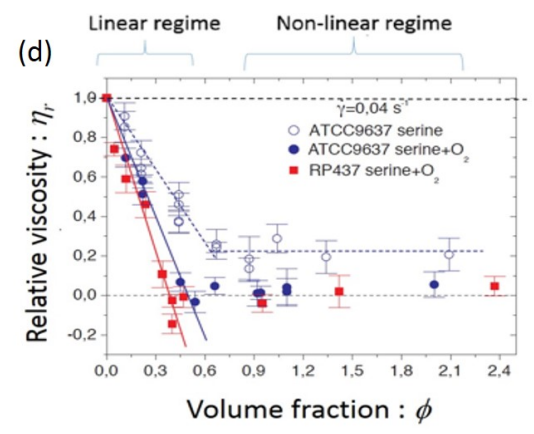

(e)

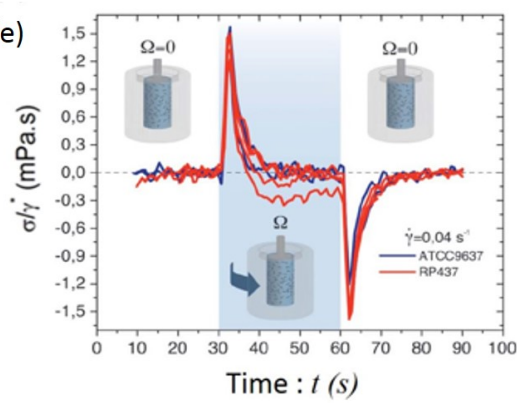

Fig. 5. Low shear rheometry [19]. (a) schematics of the Couette-shear apparatus (b) Response of an E.coli suspension $\left(\phi=2.010^{3}\right.$ to different shear rates $\dot{\gamma}$. (c) Rheogram for the relative viscosity : $\eta / \eta_{0}$ vs $\dot{\gamma}$ at different concentrations $\phi$. (d) Linear and non-linear viscous response for two strains of E.coli (ATCC9637 and RP437) [19]. The active plateau relative viscosity $\eta_{p} / \eta_{0}$ is displayed as a function of the concentration $\phi$. (e) Viscous response at $\dot{\gamma}=4.010^{-2} s^{-1}$ for ATCC9637 and RP437 in the non-linear regime.

to the suspension. This is an amino-acid allowing the bacteria to swim in absence of oxygen $[57,58]$. Thus a good and constant activity level can be kept for at least half an hour in a large concentration range. Note that the $V_{0}$ and $D_{t}$ values are lower than the corresponding values in presence of $\mathrm{O}_{2}$. The results presented in the following, were obtained with two wild type E.coli (ATCC9637 and RP437). These strains have slightly different shapes and activities.

\subsection{Low-shear rheometry}

In Lopez et al. [19], to assess the full reological response of E.coli suspensions, rheometric measurements were performed in a low-shear Taylor-Couette shear device. To be able to reach the active plateau, one has a-priori to go to very low shear rates ( $P e<1$ implies $\dot{\gamma}<0.1 s^{-1}$ ) and measure viscosities of the order or less than the viscosity of water $\left(10^{-3}\right.$ Pa.s $)$. This is not possible with conventional rheometers. The configuration of a low-shear viscometer allowing such measurements, is an external cylinder that rotates at an angular rotation rate $\Omega$. The inner cylinder is suspended via a torsion wire and its angular position is maintained constant via a feedback loop by exerting a compensating torque. Due to the feedback, the torque exerted on the inner cylinder is obtained with very high precision. The gap is $h=500 \mu m$ and therefore at constant $\Omega$, the shear rate is homogeneous and very well defined (for an 
homogeneous viscous fluid). Given a shear stress $\sigma$ measured for an imposed shear rate $\dot{\gamma}$ an effective viscosity $\eta=\sigma \dot{\gamma}$ is then obtained.

\subsection{Viscous response}

For the suspending fluid alone (a Newtonian fluid, $\eta_{0}=1.4 \mathrm{mPa} . \mathrm{s}$ ), stress timeresponses at the start or at the stop of the applied shear are fast and correspond to the device compliance (grey and black lines in Fig.5b). A similar behaviour is observed for the bacterial suspensions probed at high shear rates which moreover displays a viscosity higher than $\eta_{0}$ as generically observed for passive particles suspensions. But for low shear rates, a strikingly different behaviour shows up. When shear starts, the stress jumps to the value measured in the absence of bacteria and then, after an exponential decrease lasting a few seconds, it reaches a steady effective viscosity. When shear stops, the stress decreases abruptly to eventually change sign. In this last case, the bacteria motion induces a motive stress on the Taylor- Couette device. Thereafter, the trajectory randomization processes affect the swimming direction and stress relaxes exponentially to 0 . In first approximation, the characteristic time $\tau_{r}$ can be interpreted as stemming from a rotational diffusion of the bacteria orientation with a coefficient such as $D_{r}=\frac{1}{2 \tau_{r}}$ [28]. Here $\tau_{r} \simeq 2.4 \pm 0.3 s$ corresponding to a rotational diffusion value $\left(D_{r} \approx 0.5 r d^{2} / s\right)$ smaller than what has been currently reported for wild-type E.coli undergoing run-tumble motion [28].

Fig.5c displays a full rheogram i.e. the suspension viscosity $\eta$ as a function of $\dot{\gamma}$ for different volume fractions ranging from $\phi=0.11 \%\left(1.1 \times 10^{9}\right.$ bact $\left./ \mathrm{mL}\right)$ up to $\phi=0.67 \%\left(6.7 \times 10^{9} \mathrm{bact} / \mathrm{mL}\right)$. At low shear rate the "active viscous plateau" $\eta_{p}(\phi)$ appears. As predicted, $\eta_{p}(\phi)$ decreases linearly with the concentration (see Fig.5a in the linear regime).

\subsection{The non-linear and semi-dilute regime}

When the bacteria volume fraction is further increased, viscosity becomes independent of the concentration (Fig.5d). This is suggesting a transition between a dilute and a semi-dilute regime. Indeed, this last regime is not predicted by dilute suspension theories for which the viscous response should remain linear in concentration. Nonlinear effects may come into play as the flow induced by the swimming bacteria may strongly influence the mean shear flow responsible for the bacteria orientation. It is also a regime where clustering interaction leading to collective motion could come significantly into play [16]. For the ATCC9637 strain in a medium that doesn't contain oxygen, this regime appears at $\phi>0.7 \%$ and $\eta_{p} / \eta_{0} \sim 0.2$. In oxygenated conditions and for highly motile strains, the viscosity $\eta_{p} / \eta_{0}$ reaches a constant value $\sim 0$ for $\phi \sim 0.5 \%$ and $\phi \sim 0.4 \%$ for ATCC9637 and RP437 respectively. It means that over a significant range of concentration, one obtains a viscous response such that the local viscous dissipation is macroscopically entirely compensated by the swimming activity.

\subsection{Microfluidic rheometry}

The previous measurements where made in a device producing a simple shear (at least for the dilute case). In a confined environment as a micro-fluidic Hele-shaw channel, the basic flow profile is parabolic and the question is whether an active viscous response as described before, can also manifest itself at low shear rate. To 
(a)

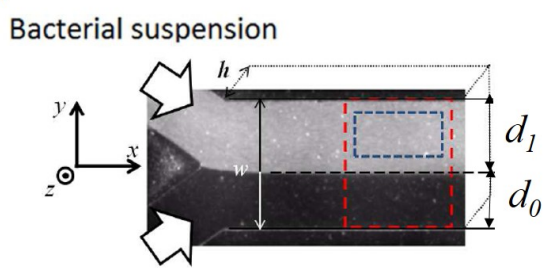

Suspending fluid

(c)

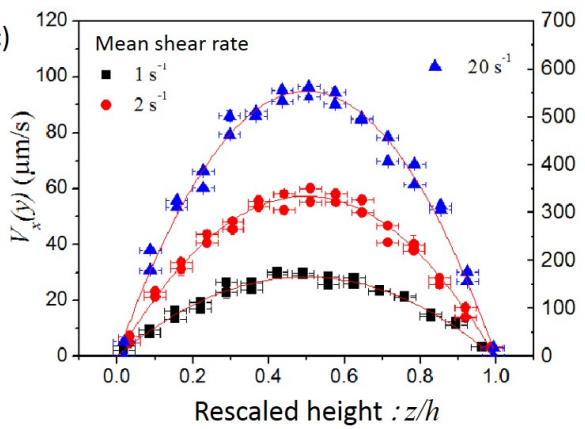

(b)

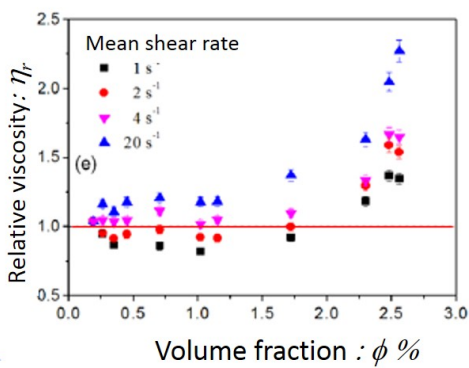

(d)

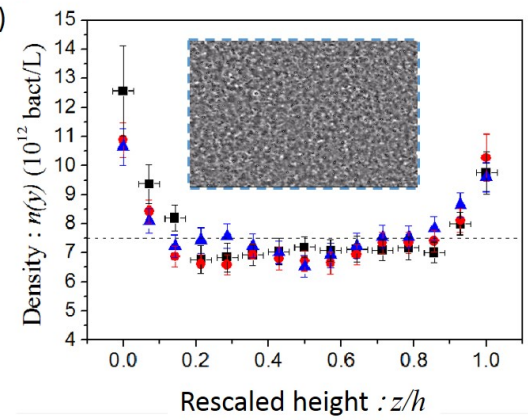

Fig. 6. Micro-fluidic rheometry of a wild-type ATCC9637 E.coli suspension( see [18]). (a) Sketch of the set-up and methodology to extract the interface position. (b) Relative viscosity as a function of volume fraction $\phi$ for different shear rate values. (c) Flow profile $V_{x}(z)$, obtained by tracking passive $2 \mu m$ latex spheres at different flow rates $Q$. Bacteria concentration profile $n(z)$ at different flow rates $Q$.

probe the emergence of such an "active viscosity", a micro-fluidic system initially proposed by Guillot and collaborators [60], was built. These authors have shown that a Y shape micro-fluidic channel is well suited to compare the viscosities of two simple liquids at low shear. An adaptation of such a device to bacterial fluids was done by Gachelin et al. [18] (see Fig 6). On one arm, the active suspension is injected and on the other arm, the suspending fluid in injected at the same flow rate $Q$. The channel thickness is $h=100 \mu \mathrm{m}$. From the position of the interface between the pure fluid and the suspension, one may extract the suspension relative viscosity. The channel widths being respectively $d_{0}$ (pure fluid) and $d_{1}$ (suspensions), one has $Q=\frac{h^{3} d_{0}}{12 \eta_{0}} \partial P / \partial x==$ $\frac{h^{3} d_{1}}{12 \eta} \partial P / \partial x$. Thus, by measuring the interface position, homogeneous in $x$, so that the pressure gradients are identical on both sides, the relative viscosity is obtained as:

$$
\frac{\eta}{\eta_{0}}=\frac{d_{1}}{d_{0}}
$$

This measured value of relative viscosity can be associated with a shear rate : $\dot{\gamma}_{M}=$ $6 Q / h^{2} d_{1}$. As displayed in fig.6, the effective viscosity rheogram shows a non-Newtonian viscosity. Varying the bacteria density and the flow rate, one can observe a regime specific to active fluids, where the relative viscosity is lower than the viscosity of the suspending viscous fluid. Such micro-fluidic devices also have the advantage of allowing a direct visualization of the bacteria in the fluid. Using a fast camera coupled to the microscope, the vertical profiles for the bacteria density and the corresponding velocity distributions for both the fluid (tracking passive tracers) and the bacteria, can be obtained. The measurements show that the basic flow profile is not significantly affected by the non-Newtonian character of the active suspensions. However, 
the technical limitations due to the dynamical stability of syringes undergoing stickslip motion at low flow rates, has prevented so far to explore the very low shear-rate regime.

\section{Conclusion}

In conclusion, the aim of this paper was to establish an elementary connection between a a simple vision of active fluids statistical mechanics (a "bacterial gas") under flow and recent experiments with bacteria suspensions. Interestingly, in the dilute limit, a good agreement between model predictions and experimental measurements was reached. The viscosity rheograms come out as similar. At low-shear, the viscosity is found to decrease with concentration at values lower than the suspending fluid viscosity. It is also interesting that such a peculiar behaviour can be found more generally in microfluidic geometries where the shear-rate is not uniform. The most striking feature of the experimental rheological response is indeed the emergence of a viscousless "superfluidity" regime, in a large range of concentrations. Presently, there is no micro-macro micro-hydrodynamic calculation describing the impact of bacteria interactions and the possible influence of collective organization on the macroscopic rheology to predict such a regime. However, there are some arguments based on the emergence of coupling terms in phenomenological transport equations, pointing on the possibility of a transition to a zero-viscosity regime [61-63]. It would be interesting to confront directly these predictions, and most of all, identify precisely the spontaneous collective organization processes undertaken by the bacteria in the flow when reaching the viscousless regime. In the framework of the low shear rheometric device used by Lopez et al., it is so far impossible to visualise in-situ the organization of the bacteria. However, the fact that an active viscosity regime was already identified for a micro-fluidic channel is encouraging. The experimental challenge would be now to push the measurements towards the onset for a zero-viscosity transition if it exists in this context.

Acknowledgement To built this synthetic view, EC acknowledges enlightening scientific discussions with Prof. Rodrigo Soto at the University of Chile in Santiago. Also a large if not an essential part of this material is a tribute to the dedicated work of former PhD students: Dr Gaston Mino, Dr Jeremie Gachelin ESPCI and Dr Matias Lopez, Paris-Sud . This work is currently supported by the ANR grant "BacFlow" ANR-15-CE30-0013.

\section{References}

1. T.Vicsek et A. Zafeiris, Collective motion, Physics Reports 517, 71 (2012)

2. P.W. Anderson, Science, 177,393 (1972).

3. C. Marchetti et al. Rev.Mod.Phys.,85,1143 (2013).

4. A. Baskaran and M. C. Marchetti, Proc. Natl. Acad. Sci. U.S.A. 106, 15567 (2009).

5. X.-L. Wu and A. Libchaber, Phys. Rev. Lett. 84, 3017 (2000).

6. G. Mino et al., Phys.Rev.Lett 106, 048102 (2011).

7. L. G. Wilson et al. Phys. Rev. Lett. 106, 018101 (2011).

8. P. Galajda, J. Kleymer, P. Chaikin and R. Austin, J. Bacteriol., 189, 8704 (2007).

9. E. Hulme et al., Lab Chip, 8, 1888 (2008).

10. J. Hill, O. Kalkanci, J. L. McMurry and H. Koser, Phys. Rev. Lett.,98, 068101 (2007).

11. E. Altshuler et al., Soft-Matter, 9, 1864 (2013).

12. R. Rusconi, J. S. Guasto and R. Stocker, Nat. Phy.,10,494498 (2014). 
13. C. Dombrowski et al., Phys. Rev. Lett. 93, 098103(2004).

14. Sokolov A and Aranson I S, Phys. Rev. Lett. 109248109 (2012 ).

15. Dunkel J et al Phys. Rev. Lett. 110228102 (2013).

16. J. Gachelin,A. Rousselet, A. Lindner, E. Clement, N. J. Phys. 16, 025003 (2014).

17. A. Sokolov et al., Phys. Rev. Lett. 103, 148101 (2009).

18. J.Gachelin et al. Phys. Rev. Lett. 110, 268103 (2013).

19. Lopez H.M., J. Gachelin, C. Douarche, H.Auradou, E. Clement, Phys. Rev. Lett. 115, 028301 (2015).

20. E.M.Purcell, Am.J.Phys. 45, 11 (1997)

21. E.Lauga and T. Powers, Rep. Prog. Phys. 72096601 (2009).

22. S. Childress, Mechanics of Swimming and Flying (Cambridge University Press, Cambridge, 1981).

23. H. C. Berg, E.coli in Motion, Springer-Verlag, New York, 2004.

24. V. Martinez et al. Proc Natl Acad Sci USA, 111, 17771(2014).

25. Guasto JS, Johnson KA, Gollub JP, Phys Rev Lett 105, 168102 (2010).

26. Drescher et al. Phys Rev Lett 105(16):168101.

27. Drescher et al. Proc Natl Acad Sci USA 108, 10940 (2011).

28. J. Saragosti, P. Silberzan, A. Buguin, PLoS ONE 7, e35412 (2012).

29. V. A. Martinez et al. , Biophys J. 103: 16371647 (2012).

30. H.C. Berg and D.A. Brown, Nature 239, 500 (1972).

31. K. Son, J. S. Guasto and R.Stocker, Nature Physics 9, 494 (2013)

32. R.E. Goldstein, M.Polin, and I.Tuval, Phys Rev Lett 103, 168103 (2009)

33. S. H.Larsen, R. W.Reader, E. N.Kort, W. W.Tso, J. Adler, Nature 249, 7477 (1974).

34. M. Schnitzer, M., Phys. Rev. E, 48, 25532568 (1993).

35. M.J. Tindall, P.K. Maini, S.L. Porter, J.P. Armitage, Bulletin of Mathematical Biology 70: 15701607 (2008).

36. G.H. Wadhams, J.P. Armitage, Nature Reviews Molecular Cell Biology 5, 1024-1037 (2004)

37. Qian L et al. Phys. Fluids 19061701 (2007).

38. J. P. Hernndez-Ortiz, C. G. Stoltz, and M. D. Graham, Phys.Rev. Lett. 95, 204501 (2005).

39. Saintillan, D. and Shelley, M. J. Phys. Rev. Lett. 99, 058102 (2007).

40. D.Saintillan and M.J. Shelley J.R. Soc. Interface 9, 571 (2012).

41. Simha RA, Ramaswamy S, Physical Review Letters, 89, 058101 (2002)

42. G. Subramanian and D. L. Koch, J. Fluid Mech. 632, 359 (2009).

43. A. Sokolov, I. S. Aranson, J. O. Kessler, and R. E. Goldstein. Phys. Rev.Lett., 98:158102, (2007).

44. H. P. Zhang, A. Be'er, E.- L. Florin, and H. L. Swinney, PNAS 107, 13626 (2010).

45. D. Saintillan, M.J. Shelley C. R. Physique 14, 497 (2013).

46. A. Zöttl and H. Stark, Phys. Rev. Lett 108, 218104 (2012).

47. AP Berke, Turner L, Berg HC, Lauga E, Phys Rev Lett 101, 038102 (2008).

48. Li G, Tang JX, Phys Rev Lett 103, 078101 (2009) .

49. J. Elgeti, Gompper G, Europhys Lett 85:38002 (2009).

50. B. Ezhilan, D. Saintillan, Journal of Fluid Mechanics, 777 482-522 (2015)

51. J.Gachelin, PhD Thesis, University Pierre et Marie Curie, December 2014.

52. S. Rafaï, L. Jibuti, and P. Peyla, Phys. Rev. Lett. 104, 098102 (2010).

53. Haines B.M. et al. Phys. Rev. E 80, 041922 (2009).

54. D. Saintillan, Exp. Mech. 50, 125 (2010).

55. S.D Ryan et al., Phys. Rev. E 83, 050904(R) (2011).

56. Y. Hatwalne, S. Ramaswamy, M. Rao, and R. A. Simha, Phys. Rev. Lett. 92, 118101 (2004)

57. J. Adler, J. Bacteriol. 92, 121-129 (1966).

58. C. Douarche, A. Buguin, H. Salman and A. Libchaber, Phys. Rev. Lett. 102, 198101 (2009).

59. J. Hinch and G. Leal, J. Fluid Mech.52, 683712 (1972). 
60. P. Guillot et al., Langmuir 22, 6438 (2006); P. Galambos and F. Forster, Micro-ElectroMechanicical System (MEMS), 1998 p. 187 (unpublished); P. Nghe, P.Tabeling, and A. Ajdari, J. Non-Newtonian Fluid Mech. 165, 313 (2010).

61. M. E. Cates, S.M Fielding, D. Marenduzzo, E. Orlandini, and J. M. Yeomans, Phys. Rev. Lett. 101, 068102 (2008).

62. L. Giomi, T. B. Liverpool, and M. C. Marchetti, Phys. Rev. E 81, 051908 (2010).

63. S. Fü rthauer, M. Neef, S.W. Grill, K. Kruse, and F. Jülicher, New J. Phys. 14, 023001 (2012).

64. R. Winkler et al. EPJST, this issue (2016).

65. U.B. Kaupp et al. EPJST, this issue (2016).

66. M. Polin et al. EPJST, this issue (2016).

67. D. Marenduzzo et al. EPJST, this issue (2016). 